\title{
Modulatory molecules involved in sleep
}

\author{
Luis D. Nieblas-Beltrán, Edel A. Hernández-González and Alberto K. De la Herrán-Arita* \\ Laboratory of Neuroscience, School of Medicine, Autonomous University of Sinaloa, Culiacán, México \\ Fraternidad Corintios A.C.
}

\begin{abstract}
Sleep is a vital process to the overall health of any individual. It is fundamental for many physiological processes, and higher cognitive functions like memory, learning and emotions. In addition, it assists in some metabolic and immune processes, among other functions.

Sleep architecture is divided in two stages, in NREM and REM sleep. The difference between them is the brain activity, ocular movements, the dynamism and modulation of different systems, neurotransmitter release, and some endogenous substances.

In this paper, we will recapitulate the main modulatory molecules involved in the regulation of this complex and fundamental activity.
\end{abstract}

\begin{abstract}
Abbreviations: 5-HT: 5-Hydroxytryptamine, Ach: Acetylcholine, AMPA: Alpha-Amino-3-Hydroxy-5-Methyl-4-Isoxazolepropionic Acid, ARAS: Ascending Reticular Activating System, ATP: Adenosine Triphosphate, cAMP: Cyclic Adenosine Monophosphate, CNS: Central Nervous System, CRF: Corticotrophin Releasing Factor, DA: Dopamine, DR: Dorsal Raphe, EEG: Electroencephalography, EMG: Electromyography, GABA: Gamma-Amino Butyric Acid, GHRH: Growth Hormone-Releasing Hormone, Glu: Glutamate, GPCRs: G Protein-Coupled Receptors, HDC: L-Histidine Decarboxylase, HDL: High-Density Lipoprotein, IL-1: Interleukin-1, KA: Kainate, L- DOPA: L-3,4-Dihydroxyphenylalanine, LC: Locus Coeruleus, LDL: Low-Density Lipoprotein, LDT: Lateral Dorsal Tegmental, LH: Lateral Hypothalamus, mRNA: Messenger Ribonucleic Acid, NA: Noradrenaline, NMDA: N-Methyl-D-Aspartate, NREM: Non-Rapid Eye Movement, PGD2: Prostaglandin D2, PGs: Prostaglandins, PPT: Pedunculopontine Tegmental, PrH: Praepositus Hypoglossal, REM sleep: Rapid Eye Movement Sleep, SCN: Suprachiasmatic Nucleus, TNF- a: Tumor Necrosis Factor Alpha, TRH: Thyrotropin Releasing Hormone.
\end{abstract}

\section{Introduction}

Sleep is a highly intricate and regulated state; nevertheless, advances and development of technology in neuroscience have provided us with the necessary tools to unravel the mysteries of sleep. Modern sleep research began in 1953 when Aserinski and Kleitman discovered rapid eye movement (REM) sleep [1].

There are some behavioral features of sleep in human beings that differ from wakefulness. Among them, is the decrease of awareness and responsiveness to external stimuli, and unlike other disease states as stupor and coma, it is easily reversible with stimulation [2].

Even though there is no consensus on the function of sleep, some scientists agree that sleep is involved in many functions, including the restoration of energy, the elimination of free radicals accumulated during the day, modulation of cortical activity, thermal, metabolic and endocrine regulation, immune activation and memory consolidation $[2,3]$.

Electroencephalography (EEG) and electromyography (EMG) are useful appliances to monitor the electric cortical activity in the different phases while we are sleeping. Generally speaking, the EEG has desynchronized waves during wakefulness, slow and delta oscillations during non-REM (NREM) sleep, and desynchronized activity (similar to wakefulness) during REM sleep. The EMG indicates high muscle activity during wakefulness and a total lack of muscle tone in the NREM and REM sleep [4,5].

Several studies have elucidated the molecules that are secreted during these different states.

\section{Dopamine}

Abrupt variations in neurotransmitter levels are known to occur as the brain progresses through the sleep-wake cycle. These changes are coordinated by manifold interactions that systematically modulate the firing rate of cholinergic, orexinergic and monoaminergic neurons. These neurons, in turn, send efferent projections to cortical and subcortical structures, generating models of brain and muscle activity characteristic of the three brain states: waking, NREM sleep and REM sleep. REM sleep is also characterized by an increase in dopamine release [6,7].

Dopamine (DA) is one of the main neurotransmitters of the central nervous system (CNS). It belongs to the group of catecholamines, along with noradrenaline and adrenalin, which are derived from DA.

DA initially is formed by hidroxylation of L-tyrosine to 3,4-dihidroxi-L-phenylalanine (L-DOPA) by the action of the enzyme tyrosine hydroxylase. The conversion of L-DOPA to DA is catalyzed by the enzyme DOPA descarboxylase.

Five types of DA receptors have been described, fitted in two families, $D_{1}$ and $D_{2}$. They belong to the superfamily of $G$ proteincoupled receptors. These receptors are expressed in several areas of the

Correspondence to: Alberto K. De la Herrán-Arita, M.D, Ph.D, Laboratory of Neuroscience, School of Medicine, Autonomous University of Sinaloa, Culiacán, Sinaloa, México, Tel: +52 (667) 775.4697, E-mail: alberto.kousuke@uas.edu.mx

Received: June 20, 2016; Accepted: July 08, 2016; Published: July 12, 2016 
CNS, like the striatum, nucleus accumbens, substantia nigra, thalamus, and cortex.

Numerous studies have revealed the role of DA in the sleepwake cycle, particularly in regulating the circadian rhythm. There is an increase of DA levels during wakefulness, thus, it facilitates the wakefulness state and enables the muscle activity [1].

DA has an important role in modulating the activity in the globus pallidus. The striatum receives DA innervation from the substantia nigra pars compacta, where it interacts with $\mathrm{D}_{2}$ receptors in GABaergic striatopallidal neurons to increase the activity in the globus pallidus. Lesions in the substantia nigra result in deep insomnia in mice and cats. Conversely, systemic $\mathrm{D}_{2}$ agonist administration causes sleepiness and sleep in humans. In addition, these results suggest that substantia nigra pars compacta DA neurons projecting to the striatum play a role in sleep-wake control by disinhibiting the globus pallidus [6].

\section{Noradrenaline}

Noradrenaline (NA) is a catecholamine with multiple functions. It acts as a hormone and as a neurotransmitter.

As a stress hormone, NA stimulates parts of the brain where attention and responding actions are controlled. Along with adrenaline, NA also takes part in the fight-or-flight response.

The synthesis of NA begins with the synthesis of DA. Once DA is synthesized and stored in synaptic vesicles, an enzyme named dopamine- $\beta$-hydroxylase further hydroxylates DA into NA. The final stage of NA synthesis occurs in the synaptic vesicles.

When an action potential triggers the release of NA into the synaptic cleft, it interacts with noradrenergic receptors on the postsynaptic neuron; afterwards, a reuptake process reabsorbs remnant NA back into the presynaptic neuron. NA also can be degraded by the enzyme monoamine oxidase.

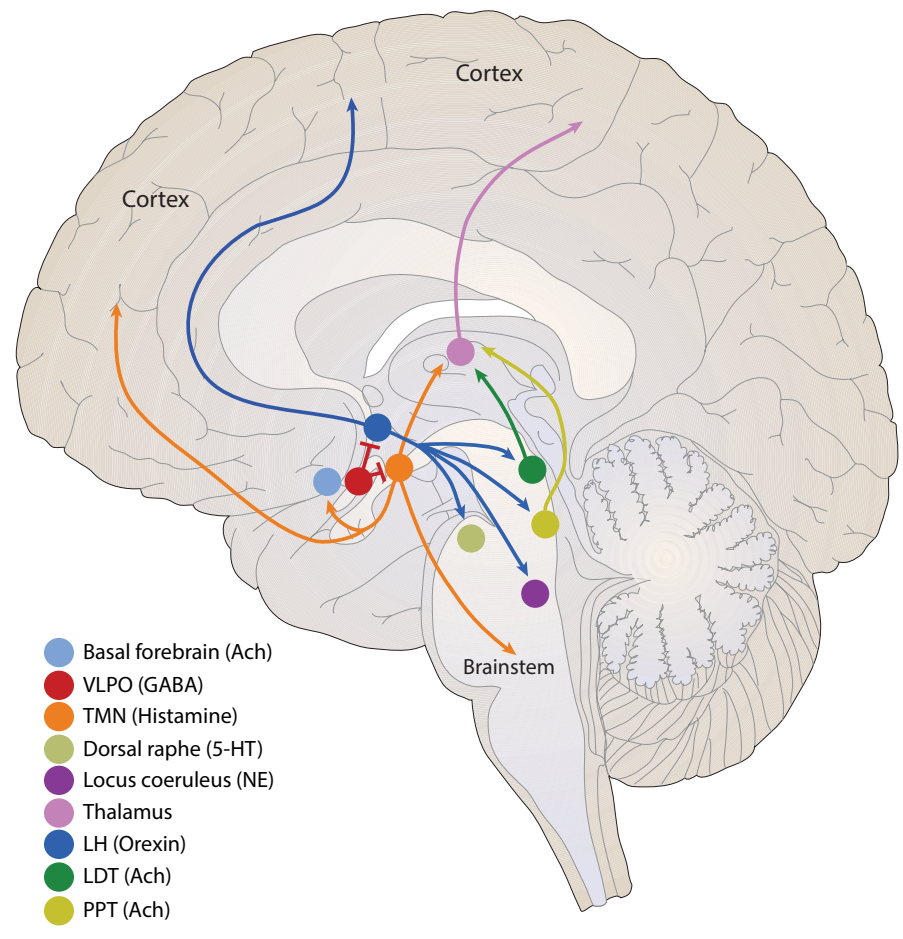

Figure 1. Main CNS nuclei that influence sleep.
Noradrenergic neurons can be found mostly in seven regions of the pons and medulla, as well as the thalamus. The most important noradrenergic system has neurons originating from the locus coeruleus (LC), a nucleus found in the dorsal pons (Figure 1).

The LC is the primary source of brain NA. NA interacts with three major receptor families, $\alpha 1, \alpha 2$, and $\beta$. $\alpha 1$ - and $\beta$-receptors are thought to reside in postsynaptic neurons, whereas $\alpha 2$-receptors are present both pre and postsynaptically. Initial electrophysiological observations indicated that the LC-noradrenergic system plays a determining role in the regulation of sleep-wake cycle. For example, LC neurons display the highest discharge rates during wakefulness and lower rates during sleep. Importantly, alterations in LC discharge rate precede changes in behavioral state. NA promotes waking acting $\alpha_{1}$ and $\beta$ receptors within medial preoptic and medial septal areas. Moreover, modulation of noradrenergic activity is essential for preservation of muscle tone during wakefulness. The activity of the LC is almost absent during REM sleep.

Some studies have shown that NA levels in ventricular cerebrospinal fluid correlate with circadian variations in primates. High levels of NA during the light hours and low during the dark hours; interestingly, the principal metabolites of NA did not exhibit variations [8].

\section{Histamine}

Histamine is an organic nitrogenous compound involved in local immune responses, physiological function in the gut, and as a neurotransmitter. Histamine is synthesized by pyridoxal phosphate (vitamin B6)-containing L-histidine decarboxylase (HDC) from the amino acid histidine. It is synthesized and released by different human cells, especially basophils, mast cells, platelets, histaminergic neurons, lymphocytes and enterochromaffin cells.

Histamine exerts its effects on target cells in various tissues by binding to four receptors $\left(\mathrm{H}_{1}, \mathrm{H}_{2}, \mathrm{H}_{3}\right.$, and $\left.\mathrm{H}_{4}\right)$. These receptors belong to the $\mathrm{G}$ protein-coupled receptors family (GPCRs). $\mathrm{H}_{1}$ and $\mathrm{H}_{2}$ are widely distributed in peripheral organs and in the $\mathrm{CNS}_{3} \mathrm{H}_{3}$ is mostly found in the CNS, and $\mathrm{H}_{4}$ receptors are found in hematopoietic cells [9].

The histaminergic system is found within the posterior hypothalamus, with projections to practically all the CNS (Figure 1). Histamine regulates the sleep-wake cycle via the $\mathrm{H}_{1}$ and $\mathrm{H}_{3}$ receptor [10], by modulating the response of various neurotransmitters (5-HT, DA, NA, and acetylcholine). The response can be either excitatory $\left(\mathrm{H}_{1}\right.$ receptor) or inhibitory $\left(\mathrm{H}_{3}\right.$ receptor). In the hypothalamus, histamine modulates the sleep-wake cycle by activating the $\mathrm{H}_{1}$ receptor, and conversely, the $\mathrm{H}_{3}$ receptor acts like an auto-receptor that diminishes the synthesis and release of histamine $[1,10]$. Histaminergic cells are active during wakefulness; however, during fatigue or periods of rest, they present a slow firing rate and cease their activity when NREM and REM phases occur [1]. In addition, the histaminergic system interacts with the orexinergic system, where histaminergic neurons send wide projections to the lateral hypothalamus, and thus promoting wakefulness [10].

\section{Serotonin}

In the CNS, serotonin (5-HT) is a monoamine neurotransmitter synthesized in the dorsal raphe (DR) nuclei. The DR is part of the reticular formation, which is located in both the pons and the medulla (Figure 1). It projects diffusely throughout the brain, having a strong effect in practically the entire CNS when activated. 
Tryptophan is the precursor of 5-HT. Tryptophan is taken up by serotonergic neurons in restricted brain areas like the DR. Once it enters the neurons, an enzyme called tryptophan hydroxylase adds the hydroxyl group and produces 5-HTP (5-hydroxytryptophan). 5-HTP is further decarboxylated by aromatic L-amino acid decarboxylase to produce 5-HT. 5-HT is then stored in synaptic vesicles and docked at the nerve terminals, where it awaits an action potential. Release of 5-HT into the synaptic cleft activates serotonergic receptors in the post-synaptic neurons.

There are seven types of 5 -HT receptors $\left(5-\mathrm{HTP}_{1}-5-\mathrm{HTP}_{7}\right)$. All of them are G- protein coupled receptors, except $5 \mathrm{HTP}_{3}$, which is an ionotropic receptor [1].

5-HT has been implicated as a key player in sleep regulation; it has been hypothesized that 5-HT stimulates the liberation of sleep-induced substances, facilitating the initiation of NREM sleep [8]. Administration of L-tryptophan induces sleep onset, acting like a natural hypnotic; in addition, it also decreases sleep latency and nocturnal awakenings [1]. Lack of L-tryptophan is associated with a decrease of REM sleep. 5-HT release spikes down swiftly at the beginning of REM sleep, and rapidly increases with the subsequent NREM sleep [8].

In mice, 5-HT decreases the amount of NREM sleep; conversely, in humans, it reduces the amount of REM sleep without affecting NREM sleep. In animal models, administration of 5-HT synthesis causes acute insomnia.

Lesions in the DR induce a shortfall of 5-HT, resulting in insomnia. This can be reversed with 5-HTP administration. 5-HT levels are variable throughout the day. Maximum levels occur during the day and decrease at night; however, receptor affinity is higher during the night [1].

\section{Acetylcholine}

Acetylcholine (Ach) is a neurotransmitter that has been implicated with cognitive processing, arousal, and attention in the brain.

In the CNS, there are two major subtypes of Ach receptors, the metabotropic muscarinic receptors and the ionotropic nicotinic receptors. Both share the property of being activated by the endogenous neurotransmitter Ach. Activation of muscarinic receptors is relatively slow (milliseconds to seconds) and, depending on the subtypes $\left(\mathrm{M}_{1}-\right.$ $\mathrm{M}_{5}$ ), they directly remodel cellular homeostasis of phospholipase C, inositol trisphosphate, cAMP, and free calcium. Some studies have shown that the $\mathrm{M}_{2}$ receptor promotes REM sleep [11].

Ach plays an important role in wakefulness, it is related with REM sleep maintenance, thalamic state of alertness, as well as striatum motor activity control. In cats, cortical release of Ach is higher during wakefulness [1]. Recent studies with optogenetics have shown that stimulation of the basal forebrain cholinergic neurons increase the transitions between NREM sleep and wakefulness [12]. In cats, lesions of the dorsolateral pons induce an increase of REM sleep without atonia; they present a prey-catching behavior, as if they were acting out their dreams. In addition, cholinergic dorsolateral pontine neurons project to the ventral medulla, where they define synapses with inhibitory neurons that project and inhibit spinal motor neurons; thereby, impeding or preventing muscle movement during REM sleep. This inhibition is thought to be mediated by glycinergic and GABAergic mechanisms [13].

\section{Glutamate}

Glutamate (Glu) is the major excitatory neurotransmitter in brain, acting through ionotropic receptors: N-methyl-D-aspartate (NMDA), alpha-amino-3-hydroxy-5-methyl-4-isoxazolepropionic acid (AMPA) and Kainate (KA). In addition, it also acts through metabotropic receptors. Glu receptors play a vital role in the mediation of excitatory synaptic transmission. These are found throughout the brain and spinal cord, both in neurons and glia. As an amino acid and neurotransmitter, Glu has a large array of normal physiological functions [14].

Glu activity regulates the sleep-wake cycle by activating the ascending reticular activating system (ARAS) and the suprachiasmatic nucleus.

Glutamatergic neurons within the hypothalamus are activated by orexin, and they, in turn, stimulate the orexin neurons to command the hypothalamic arousal system [15].

\section{GABA}

The $\gamma$-aminobutyric acid (GABA) is the most important inhibitor neurotransmitter in the CNS. GABA synthesis begins with glutamate breakdown by the enzyme glutamic acid decarboxylase (GAD) and vitamin $\mathrm{B}_{6}$ as cofactor [16]. When GABA is released, it must interact with specific receptors. There are three GABA receptors: $G_{A B A}, G_{A B A}$ and $\mathrm{GABA}_{c}$. The receptor involved in sleep induction is $\mathrm{GABA}_{\mathrm{A}}$. It is an ionotropic receptor that allows the entry of $\mathrm{Cl}$-, improving a neuronal hyperpolarization, and thus, inhibiting neurotransmission [17]. At the end of its action, GABA disengages the receptor and it is reuptake by some neurons and neuroglia to be degraded by GABA transaminase.

Unlike wakefulness, during REM sleep there is an increase in the release of GABA from GABAergic neurons in different areas of the CNS. Anatomical and electrophysiological evidence has demonstrated that the primary GABAergic input to the LC derives from the praepositus hypoglossal (PrH) nucleus of the medulla [18]. Besides the LC, GABAergic neurons are intermingled with glutamatergic and cholinergic neurons in the pedunculopontine tegmental (PPT) and lateral dorsal tegmental (LDT) nuclei. Some studies demonstrated that injections of GABA in the PPT induce REM sleep [19].

\section{Adenosine}

Adenosine is an endogenous sleep-promoting substance formed by the junction of adenine and ribose. It derives from ATP degradation in glial synaptic vesicles. A balance coexists between the extracellular and intracellular adenosine, mediated by transporters in the brain tissue [20].

There are four adenosine receptors: $A_{1}, A_{2 A}, A_{2 B} y A_{3}$ The stimulation of adenosine $A_{1}$ receptor produces a hyperpolarization and inhibits synaptic transmission. This occurs mainly in cholinergic neurons, by increasing the conductance of potassium channels [21].

Adenosine is mostly found in the magnocellular cholinergic forebrain and the brain stem (PPT and LDT) [22]. There is evidence that NREM sleep increases after administration of desoxicoformicine, an inhibitor of adenosine degradation. Furthermore, it was found in cats that an increase of adenosine perfusion in the brain reduces wakefulness [23].

\section{Orexins}

Orexins (hypocretins) are essential neuropeptides for an adequate wakefulness and energy balance. There are two kinds of orexins: orexin 
A (hypocretin-1) and orexin B (hypocretin-2), and are exclusively synthesized in the lateral hypothalamus (LH) (Figure 1), derived from a single protein precursor named prepro-orexin. [24]. Two cloned orexin receptors OX1R and OX2R are serpentine G-proteincoupled receptors, both of which bind orexins and are coupled to $\mathrm{Ca}^{2+}$ mobilization. Orexins neurons project within the LH throughout the central nervous system to nuclei involved in the control of feeding, sleep-wakefulness, neuroendocrine homeostasis, and autonomic regulation [25].

Orexin neurons are active during wakefulness, as indicated by the expression of Fos, and extracellular concentrations of orexin are higher during periods of wakefulness. Electrophysiological recordings from the orexin neuron region identified many wake-active neurons, with particularly high firing rates when an animal is physically active.

In vitro, orexin excites the LC and other aminergic neurons. In vivo, injections of orexin into the lateral ventricles or near specific arousal regions such as the LC increase wakefulness and markedly suppress REM sleep [26]. It is known that an orexin dysfunction or a destruction of their somas may cause the sleep disorder known as narcolepsy $[27,28]$.

\section{Melatonin}

Melatonin is an endogenous hormone produced in the pineal gland. It is derived from the amino acid tryptophan and 5-HT. This hormone is not stored, but it is released once it is synthesized. Melatonin production begins with the stimulation of the suprachiasmatic nucleus (SCN) in the anterior hypothalamus; the retina triggers this mechanism during darkness. Afterwards, the SCN activates the superior cervical ganglion and the pineal gland. This mechanism is suppressed by light [29].

The melatonin receptors are $\mathrm{ML}_{1}$ and $\mathrm{ML}_{2} . \mathrm{ML}_{1}$ receptors have more affinity to melatonin and are better distributed than $\mathrm{ML}_{2}$ They are divided in $\mathrm{ML}_{1 \mathrm{a}}$, responsible of pacing the circadian rhythm, and $\mathrm{ML}_{1 \mathrm{~b}}$, a mediator in the retina. Besides the $\mathrm{SCN}$ and retina, these receptors are also expressed in the pars tuberalis of the hypophysis, which is involved in prolactin liberation. They are also present in cerebral arteries, where they regulate the temperature and metabolite exchange [30].

Recently, studies have shown that people who are often exposed to light during the night develop a circadian dysregulation and a decrease of melatonin [31]. This can be counteracted with exogenous melatonin administration [32].

\section{Cytokines}

Cytokines are glycoproteins produced by leukocytes, and they take part in processes that regulate immunity, inflammation and hematopoiesis. They regulate a great number of physiological and pathological functions, including innate immunity, acquired immunity, and a plethora of inflammatory responses. Generally, their secretion is a brief, self-limited event. Cytokines often have multiple effects on the same target cell and may induce or inhibit the synthesis and effects of other cytokines. After binding to specific receptors on the cell surface of the target cells, cytokines produce their specific effects. Numerous signals regulate the expression of cytokine receptors. The target cells respond to cytokines by new mRNA and protein synthesis, which results in a specific biological response.

Interleukin-1 (IL-1) and tumor necrosis factor alpha (TNF- $\alpha$ ) play an important role in both innate and adaptive immunity and are crucial mediators of the host inflammatory response in natural immunity. The major cell source of IL-1 is the activated mononuclear phagocyte. The major cell source of TNF- $\alpha$ is the macrophage, specifically the endotoxin-activated mononuclear phagocyte.

IL-1 and TNF- $\alpha$ rise during NREM sleep under physiological and inflammatory conditions. Other cytokines are also likely implicated, but evidence is deficient to assume that they are sleep regulatory substances. Several of the symptoms induced by sleep loss, sleepiness, fatigue, unsatisfactory cognition, enhanced sensitivity to pain, can be caused by injection of exogenous IL-1 or TNF- $\alpha$ [33].

Observations in animals showed that injection of exogenous low doses of IL-1 or TNF-a improved NREM sleep. Conditions that intensify endogenous production of IL-1 or TNF- $\alpha$, excessive food consumption or infectious disease, promote NREM sleep. In contrast, inhibition of endogenous IL-1 or TNF- $\alpha$, using antibodies or endogenous inhibitors, inhibits unbidden sleep. These inhibitors of IL- 1 and TNF- $\alpha$ also inhibit sleep recovery after sleep deprivation. It is noted that microinjections of TNF- $\alpha$ into the anterior hypothalamus magnifies NREM sleep. IL-1 accentuates the firing rate of hypothalamic sleep-active neurons, while it inhibits wake-active neurons. Some hypothalamic neurons receptive for GHRH are also receptive for IL-1. This evidence proposes that the NREM sleep regulatory system is receptive to IL-1 and TNF- $\alpha$ [33].

Both IL-1 and TNF- $\alpha$ are influenced by several neurotransmitter systems implicated in the promotion of wakefulness. For instance, an injection of IL-1 into the LC, or the DR causes NREM sleep. Concretely, these and other information mean that IL- 1 and TNF- $\alpha$ are engaged in physiological NREM sleep regulation as well as variations in sleep associated with illness.

Both IL-1 and TNF- $\alpha$ have been associated to a numerous of clinical conditions affecting sleep disturbances. For instance, high levels of TNF- $\alpha$ are present in patients with chronic fatigue syndrome, chronic insomnia, sleep apnea, alcoholism, influenza virus infections, and rheumatoid arthritis [13,33].

\section{Peptides}

Besides orexin, there are other peptides participating in sleep regulation. Corticotrophine releasing factor (CRF) and adenocorticotrophic hormone levels during the day, but they probably participate in the activation of orexinergic neurons [34].

Thyrotropin releasing hormone (TRH) increases during wakefulness and decreases during sleep. It also has been shown to suppress cataplexy, the main symptom of narcolepsy [35].

Some peptides also improve sleep. For instance, cholecystokinin-8 increases NREM sleep by inhibiting 5-HT action ${ }^{[36]}$. Prolactin and the vasoactive intestinal peptide are REM sleep-promoting agents [36].

\section{Prostaglandin $\mathrm{D}_{2}$}

Eicosanoids are 20 -carbon fatty acids that are produced in a variety of tissues and that mediate an array of physiologic and pathologic processes.

Prostaglandins (PGs) consist of an oxygenated cyclopentane/ pentane ring with a heptanoic acid side, and an octenol side chain on adjacent carbon atoms of the ring. Each PG differs from the others in the substitution pattern of the cyclopentane ring and the side chains. These differences are responsible for the different biologic activities of the members of the PG group. 
Studies have shown that an intracerebral administration of $\mathrm{PGD}_{2}$ promotes sleep, basically NREM sleep in animals like mice and monkeys. Furthermore, inhibition of $\mathrm{PGD}_{2}$ synthesis suppresses sleep. $\mathrm{PGD}_{2}$ acts on the ventral surface of the rostral basal forebrain, outside the brain parenchyma, a sleep-promoting zone [13].

\section{Gonadal steroids}

Steroid hormones are derived from cholesterol. Cholesterol is synthesized de novo from acetate $(\sim 90 \%)$ or taken from the diet $(\sim 10 \%)$. Cholesterol is primarily produced and stored in the liver, and is transported to the cells in the form of high-density lipoprotein (HDL) and low-density lipoprotein (LDL).

Evidence from animals as well as humans, proposed an important regulation of sleep-wake cycle by ovarian steroids in females and testicular steroids in male mice. Clinical studies have confirmed that a fluctuating ovarian hormonal influences sleep throughout a woman's lifespan [37].

Rodent studies using EEG to record sleep showed that acute exogenous administration of estradiol to female rodents decreased total sleep time, but discrepancies existed as to its specific influence on REM and NREM sleep. In addition, REM sleep is hardly reduced and REM sleep episodes are shorter in the luteal phase. Higher progesterone and estradiol levels correlate with less REM sleep, a finding consistent with observations in the gonadal intact female rats although rats show a corresponding increase in wakefulness not seen in humans. Estrogen and progesterone receptors are localized in many sleep-wake regulatory nuclei, including the basal forebrain, hypothalamus, DR, and LC.

Conversely, there is a connection between testosterone levels and sleep. Androgen receptors are numerous in the suprachiasmatic nucleus and androgenic effects are important to the circadian timing system. Strong evidence shows that androgens act directly in the suprachiasmatic nucleus to modulate circadian rhythm. In male mice, testosterone is widely aromatized to estradiol in the brain; thus, estrogenic influences modulate changes in the circadian rhythm [37].
The suprachiasmatic nucleus of mammals, including humans, contains gonadal steroid receptors for progesterone, estrogens, and androgens; in other words, gonadal hormones probably affect the circadian rhythm and sleep via direct regulation of suprachiasmatic nucleus functions. Estradiol may exert its effect on circadian rhythm via extra-suprachiasmatic nucleus sites projecting to the suprachiasmatic nucleus $[17,37]$.

\section{Lactoferrin}

Lactoferrin is a no-heme glycoprotein member of the transferrin family. These proteins bind and transfer iron ions. Lactoferrin is present in several exocrine fluids and it has an important role due to its antimicrobial activity.

Some studies in patients with Parkinson's disease and REM sleep disorders showed high levels of iron, transferrin and lactoferrin in cerebrospinal fluid. Nevertheless, serum transferrin was decreased [38].

In Parkinson's disease, there is an abnormal storage of iron in the substantia nigra, one of the main sources of DA. This produces degeneration of dopaminergic neurons [39]. Furthermore, iron and other substances are liberated from dead neurons causing more damage to the healthy neurons.

The amounts of sleep can interfere with lactoferrin levels. Lactoferrin production is increased after acute exercise and after a sleep deprivation [40].

\section{Discussion}

Our knowledge of the human brain has grown enormously in the last decades. Sleep research is an intricate area; unraveling and identifying the mechanisms involved in the regulation of the sleepwake cycle has been a difficult attempt, due its nature as an intricate system. A giant leap in the way to unravel the mystery of sleep was taken with the finding of the molecules described above (Table 1), and its association with sleep-wake cycle regulation.

Table 1. Modulatory molecules involved in sleep.

\begin{tabular}{|c|c|c|}
\hline Molecule & Feat & Site of action \\
\hline Dopamine & Wakefulness & Substantianigra and striatum \\
\hline Adrenaline & Wakefulness & Locus coeruleus \\
\hline Serotonin & Wakefulness & Raphe nuclei \\
\hline Histamine & Wakefulness & Tuberomammillary nucleus \\
\hline Glutamate & Wakefulness & Suprachiasmatic nucleus \\
\hline Acetylcholine & Wakefulness & Dorsolateral pont \\
\hline GABA & Sleep & Tuberomammillary nucleus \\
\hline Adenosine & Sleep & Laterodorsal and pedunculopontine tegmental \\
\hline Orexin & Wakefulness & Tuberomammillary nucleus \\
\hline Melatonin & Sleep & Suprachiasmatic nucleus \\
\hline CRF & Wakefulness & Hypothalamus and pituitary gland \\
\hline Adenocorticotrophine & Wakefulness & Pituitary gland \\
\hline TRH & Wakefulness & Hypothalamus and pituitary gland \\
\hline Cholecystokinin & Sleep & Hypothalamus \\
\hline Prolactin & Sleep & Dorsal raphe and locus coeruleus \\
\hline Vasoactive intestinal peptide & Sleep & Hypothalamus \\
\hline Prostaglandin $\mathrm{D}_{2}$ & Sleep & Ventral surface of the rostral basal forebrain \\
\hline Interleukine-1 & Sleep & Hypothalamus, locus coeruleus, dorsal raphe \\
\hline TNF $\alpha$ & Sleep & \multirow{4}{*}{$\begin{array}{l}\text { Basal forebrain, hypothalamus, dorsal raphe nucleus, locus coeruleus and } \\
\text { suprachiasmatic nucleus }\end{array}$} \\
\hline Estradiol & \multirow[t]{3}{*}{ Probably wakefulness } & \\
\hline Estrogen & & \\
\hline Testosterone & & \\
\hline
\end{tabular}


The neural bases of sleep and wakefulness are highly related to the orexinergic, cholinergic, monoaminergic and glutamatergic systems. The activity of these systems promotes wakefulness. The orexinergic system seems to take a particular role in the promotion of wakefulness and suppression of REM sleep by giving excitatory input to the monoaminergic and cholinergic systems. Conversely, the GABAergic system in the preoptic area of the hypothalamus and brainstem promotes sleep, as well as other endogenous substances like melatonin and adenosine.

Sleep is a dynamic state with a cyclic alternation between NREM and REM sleep. The pons is critical for generating the multiple components like EEG synchronization, eye movements, muscle atonia and others characteristics of REM sleep. The sleep-wake cycle is arranged by an interaction between the circadian pacemaker located in the hypothalamic suprachiasmatic nucleus and a sleep homeostatic system whose anatomic location has been well identified (Figure 1).

Numerous neurotransmitter systems contribute to the promotion of wakefulness; however, none of them appears to be absolutely essential, though the biochemical mechanisms responsible for sleep regulation are very complex. Interpreting physiological sleep and sleep disturbances occurring during pathology is possible within the context of the brain neurotransmitters network, although it is still incomplete. Further clarification of the mechanisms responsible for the orchestration of the sleep-wake cycle still needs to be determined; this may shed light on new tools for diagnosing and treating sleep diseases and will have important implications for the comprehension of the CNS.

\section{References}

1. Negriollo, A (2013) Biochemical basis involved in sleep regulation. Arch Neurocien (Mex).

2. Carrillo P, Ramírez J, Magaña K (2013) Neurobiology of sleep and its importance: the anthology for the college student. Journal of the Faculty of Medicine of the UNAM.

3. Walker MP (2009) The role of sleep in cognition and emotion. Ann N Y Acad Sci 1156: 168-197. [Crossref]

4. Susmakova K (2004) Human Sleep and Sleep EEG. Measurement Science Review.

5. Lee SH, Dan Y (2012) Neuromodulation of brain states. Neuron 76: 209-222. [Crossref]

6. Qiu M, Yao Q, Vetrivelan R, Chen M, Lu J (2014) Nigrostriatal Dopamine Acting on Globus Pallidus Regulates Sleep. From Oxford web site: [http://cercor.oxfordjournals. org/content/early/2014/10/14/cercor.bhu241.full.pdf].

7. Dzirasa K, Ribeiro S, Costa R, Santos LM, Lin SC, et al. (2006) Dopaminergic Control of Sleep-Wake States. J Neurosci 41: 10577-10589. [Crossref]

8. Martínez J and Santamaria, J (2005) CSF markers in sleep neurobiology. Clin Chim Acta 362: 12-25. [Crossref]

9. Berridge C (2007) Noradrenergic modulation of arousal. Brain Res Rev 58: 1-17. [Crossref]

10. Thakkar M (2010) Histamine in the regulation of wakefulness. Sleep Med Rev 15: 6574. [Crossref]

11. Albuquerque E, Pereira E, Alkondon M, Rogers S (2009) Mammalian Nicotinic Acetylcholine Receptors: From Structure to Function. Physiol Rev 89: 73-120. [Crossref]

12. Zant J, Kim T, Prokai L, Szarka S, McNally J, et al. (2006) Cholinergic Neurons in the Basal Forebrain Promote Wakefulness by Actions on Neighboring Non-Cholinergic Neurons: An Opto-Dialysis Study. J Neurosci 36: 2057-2067. [Crossref]

13. Schwartz MD, Kilduff TS (2015) The Neurobiology of Sleep and Wakefulness. Psychiatr Clin North Am 38: 615-644. [Crossref]

14. Watson C, Lydic R, Baghdoyan H (2011) Sleep duration varies as a function of glutamate and GABA in rat pontine reticular formation. $J$ Neurochem 118: 571-580. [Crossref]

15. Lee SH, Dan Y (2012) Neuromodulation of brain states. Neuron 76: 209-222. [Crossref]

16. Martin DL, Rimvall K (1993) Regulation of gamma-Aminobutyric Acid Synthesis in the Brain. J Neurochem 60: 395-407 [Crossref]

17. Greene RW, Frank MG (2010) Slow wave activity during sleep: functional and therapeutic implications. Neuroscientist 16: 618-633. [Crossref]

18. Nitz D, Siegel JM (1997) GABA release in the locus coeruleus as a function of sleep/ wake state. Neuroscience 78: 795-801. [Crossref]

19. Gottesmann C (2002) GABA mechanisms and sleep. Neuroscience 111: 231-239. [Crossref]

20. Hong ZY, Huang ZL, Qu WM, Eguchi N, Urade Y, et al. (2005) An adenosine A2A receptor agonist induces sleep by increasing GABA release in the tuberomammillary nucleus to inhibit histaminergic systems in rats. J Neurochem 92: 1542-1549. [Crossref]

21. Bertorelli R, Ferri N, Adami M, Ongini E (1996) Effects of Selective Agonists and Antagonists for A1, or A2, Adenosine Receptors on Sleep-Waking Patterns in Rats. Drug Development Research.

22. Basheer R, Strecker RE, Thakkar MM, McCarley RW (2004) Adenosine and sleepwake regulation. Prog Neurobiol 73: 379-396. [Crossref]

23. Satoh S, Matsumura H, Koike N, Tokunaga Y, Maeda T, et al. (1999) Regiondependent difference in the sleep-promoting potency of an adenosine A2A receptor agonist. Eur J Neurosci 11: 1587-1597. [Crossref]

24. Yoshida Y, Fujiki N, Nakajima T, Ripley B, Matsumura H, et al. (2001) Fluctuation of extracellular hypocretin-1 (orexin A) levels in the rat in relation to the light-dark cycle and sleep-wake activities. Eur J Neurosci 14: 1075-1081. [Crossref]

25. Burdakov D, Liss B, Ashcroft FM (2003) Orexin Excites GABAergic Neurons of the Arcuate Nucleus by Activating the Sodium-Calcium Exchanger. J Neurosci 23: 49514957. [Crossref]

26. Kiyashchenko L, Mileykovskiy BY, Maidment N, Lam HA, Wu MF, et al. (2002) Release of hypocretin (orexin) during waking and sleep states. $J$ Neurosci 22: 5282 5286. [Crossref]

27. Nishino S, Mignot E (2002) Article reviewed: Plasma orexin-A is lower in patients with narcolepsy. Sleep Med 3: 377-378. [Crossref]

28. Martynska L, Witort EW, Chmielowska M, Bik W, Baranowska B (2005) The physiological role of orexins. Neuro Endocrinol Lett 26: 289-292. [Crossref]

29. Montilla P, Túnez I (2007) Melatonin, Present and Future. Nova Biomedical.

30. Dubocovich ML (2007) Melatonin receptors: role on sleep and circadian rhythm regulation. Sleep Med 8: 34-42. [Crossref]

31. Blask DE (2008) Melatonin, sleep disturbance and cancer risk. Sleep Med Rev 13: 257-264. [Crossref]

32. Cajochen C, Kräuchi K, Wirz-Justice A (2003) Role of melatonin in the regulation of human circadian rhythms and sleep. J Neuroendocrinol 15: 432-437. [Crossref]

33. Krueger JM (2008) The role of cytokines in sleep regulation. Curr Pharm Des 14: 3408-3416. [Crossref]

34. Winsky-Sommerer R, Yamanaka A, Diano S (2004) Interaction between the corticotrophin-releasing factor system and hypocretins (orexins): a novel circuit mediating the stress response. J Neurosci 24: 11439-11448. [Crossref]

35. Riehl J, Honda K, Kwan M (2000) Chronic oral administration of CG-3703, a thyrotropin releasing hormone analog, increases wake and decreases cataplexy in canine narcolepsy. Neuropsychopharmacology 23: 34-45. [Crossref]

36. Kapás L, Obál F Jr, Opp MR, Johannsen L, Krueger JM (1991) Intraperitoneal injection of cholecystokinin elicits sleep in rabbits. Physiol Behav 50: 1241-1244. [Crossref]

37. Mong JA, Baker FC, Mahoney MM, Paul KN, Schwartz MD, et al. (2011) Sleep, rhythms, and the endocrine brain: influence of sex and gonadal hormones. $J$ Neurosci 31: 16107-16116. [Crossref]

38. Yu SY, Sun L, Liu Z, Huang XY, Zuo LJ, et al. (2013) Sleep disorders in Parkinson's disease: clinical features, iron metabolism and related mechanism. PLoS One 8: e82924. [Crossref] 
39. Hu Y, Yu SY, Zuo LJ, Piao YS, Cao CJ, et al. (2015) Investigation on Abnormal Iron Metabolism and Related Inflammation in Parkinson Disease Patients with Probable RBD. PLoS One 10: e0138997. [Crossref]
40. Gillum TL, Kuennen MR, Castillo MN, Williams NL, Jordan-Patterson AT (2015). Exercise, But Not Acute Sleep Loss, Increases Salivary Antimicrobial Protein Secretion. J Strength Cond Res 29: 1359-1366. [Crossref]

Copyright: (C2016 Nieblas-Beltrán LD. This is an open-access article distributed under the terms of the Creative Commons Attribution License, which permits unrestricted use, distribution, and reproduction in any medium, provided the original author and source are credited. 